\title{
Orthogonal rational functions and interpolatory product rules on the unit circle. I. Recurrence and interpolation*
}

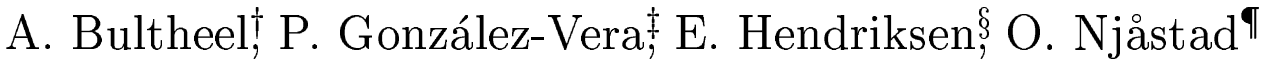

\begin{abstract}
Let $\mathcal{R}$ be the space of rational functions with poles among $\left\{\alpha_{k}, 1 / \bar{\alpha}_{k}\right\}_{k=0}^{\infty}$ with $\alpha_{0}=0$ and $\left|\alpha_{k}\right|<1, k \geq 1$. We consider sequences $\left\{\mathcal{R}_{n}\right\}_{n=0}^{\infty}$ of nested subspaces with $\cup_{n=0}^{\infty} \mathcal{R}_{n}=\mathcal{R}$. This first part will be concerned with the construction of two distinct orthogonal bases for $\mathcal{R}$. We derive an intertwined recurrence relations for these orthogonal functions which appear as denominators of certain continued fractions. By contraction of these continued fractions, these recurrences are decoupled. It is explained how, with the given problem, one can associate two sequences of interpolation data and it is shown that the approximants of the continued fractions interpolate these data in a multipoint Padé sense. In part II, interpolatory quadrature rules which are exact for all $f \in \mathcal{R}_{n}$ are constructed and their convergence is discussed as $n \rightarrow \infty$.
\end{abstract}

Keywords: orthogonal rational functions, continued fractions, rational interpolation. AMS Classification: 30B70, 33C45, 41A05

\section{Introduction}

Let $\mathbb{C}$ be the set of complex numbers and $\hat{\mathbb{C}}=\mathbb{C} \cup\{\infty\}$. We use the following notation for the unit circle and the open unit disk: $\mathbb{T}=\{z \in \mathbb{C}:|z|=1\}, \mathbb{D}=\{z \in \mathbb{C}:|z|<1\}$. The space of polynomials of degree at most $n$ is denoted as $\Pi_{n}$ and $\Pi$ is the space of all polynomials. For any pair of nonnegative integers $(m, n)$, we denote by $\Lambda_{m, n}$ the linear space of all Laurent polynomials of the form $L(z)=\sum_{j=-m}^{n} c_{j} z^{j}, c_{j} \in \mathbb{C}$, and $\Lambda$ is the space of all Laurent polynomials. For any complex function $f$, we define its substar conjugate by $f_{*}(z)=\overline{f(1 / \bar{z})}$. Let $M$ be a linear funcional defined on $\Lambda$ such that $M\{f\}$ is real whenever $f$ is real-valued and suppose that $M\{f\}>0$ whenever $f>0$. Then we can define a positive definite inner product on $\Lambda$ by

$$
\langle f, g\rangle=M\left\{f g_{*}\right\}
$$

*This research was performed as part of the European project ROLLS under contract CHRX-CT93-0416.

${ }^{\dagger}$ Department of Computer Science, K.U.Leuven, Belgium

${ }^{\ddagger}$ Department Análisis Math., Univ. La Laguna, Tenerife, Spain

$\S$ Department of Math., Univ. of Amsterdam, The Netherlands

IDepartment of Math. Sciences, Norwegian Univ. of Science and Technology, Trondheim, Norway 
For example, if $\mu$ is a positive measure on $\mathbb{T}$, then such a linear functional and the corresponding inner product are given by

$$
\int f(t) d \mu(t) \quad \text { and } \quad\langle f, g\rangle=\int f(t) \overline{g(t)} d \mu(t) .
$$

If we now orthogonalize the basis functions $1, z, z^{2}, \ldots$ for $\Pi$ with repect to this inner product, then we obtain a basis of orthogonal polynomials. In the case of the inner product (1.2) we obtain the well known polynomials orthogonal on the unit circle, studied by Szegö.

In [16], Thron has studied orthogonal Laurent polynomials on the unit circle. These are obtained by orthogonalization of the basis $1, z, z^{-1}, z^{2}, z^{-2}, \ldots$ or $1, z^{-1}, z, z^{-2}, z^{2}, \ldots$ for $\Lambda$ with respect to the inner product (1.2). These are important for the solution of trigonometric moment problems, two-point Padé approximation and quadrature on the unit circle.

Rational generalizations of the polynomial case can be obtained as follows. Let $\alpha=\left\{\alpha_{n}\right.$ : $n=0,1, \ldots\}\left(\alpha_{0}=0\right)$ be an arbitrary sequence in $\mathbb{D}$. We denote the Blaschke factor $\zeta_{k}(z)$ as

$$
\zeta_{k}(z)=z_{k} \frac{\alpha_{k}-z}{1-\bar{\alpha}_{k} z}, \quad z_{k}=\left\{\begin{array}{ll}
-1 & \text { if } \alpha_{k}=0 \\
\bar{\alpha}_{k} /\left|\alpha_{k}\right| & \text { if } \alpha_{k} \neq 0
\end{array} \quad k=1,2, \ldots\right.
$$

The Blaschke products are given by $B_{0}=1$ and $B_{k}(z)=\zeta_{1}(z) \cdots \zeta_{k}(z)$ for $k=1,2, \ldots$. These generate the spaces $\mathcal{L}_{n}=\operatorname{span}\left\{B_{0}, B_{1}, \ldots, B_{n}\right\}$ and $\mathcal{L}=\cup_{n=0}^{\infty} \mathcal{L}_{n} . \quad \mathcal{L}_{n}$ and $\mathcal{L}$ are rational generalizations of the polynomial spaces $\Pi_{n}$ and $\Pi$. Indeed, if we set

$$
\pi_{0}=1 \quad \text { and } \quad \pi_{n}(z)=\prod_{k=1}^{n}\left(1-\bar{\alpha}_{k} z\right) \quad \text { for } \quad n=1,2, \ldots
$$

then obviously

$$
\mathcal{L}_{n}=\left\{R=\frac{p_{n}}{\pi_{n}}: p_{n} \in \Pi_{n}\right\}
$$

If all $\alpha_{k}=0$, then $\mathcal{L}_{n}=\Pi_{n}$. Suppose that the linear functional $M$ is defined on the space $\mathcal{L}$ having the same properties as before. Then we can orthogonalize the basis $B_{0}, B_{1}, \ldots$ and obtain orthogonal rational functions $\phi_{n}$ such that $\mathcal{L}_{n-1} \perp \phi_{n} \in \mathcal{L}_{n}-\mathcal{L}_{n-1}$. These are the rational generalizations of the orthogonal polynomials. Since their poles are all outside the unit disk, they are analytic in $\mathbb{D} \cup \mathbb{T}$. In the case of the inner product (1.2), they are relevant for Nevanlinna-Pick interpolation, multipoint Padé approximation, and the construction of optimal rational Szegö quadrature on the unit circle. These functions were studied in the sixties by Djrbashian (see the survey [8]), Bultheel [2], and in a series of papers by the authors of which we mention only some survey papers $[3,4,6,7,5]$ which contain many references to the literature, including recent work on this topic by K. Pan and X. Li (e.g. [14, 15, 10]) and on the relation with orthogonal polynomials with respect to varying measures as discussed by G. Lopez-Lagomasino [11, 12].

In this paper, we discuss the rational generalization of the orthogonal Laurent polynomials. So, let us define $\mathcal{L}_{n *}=\left\{f: f_{*} \in \mathcal{L}_{n}\right\}=\operatorname{span}\left\{1, B_{1 *}, \ldots, B_{n *}\right\}$ and $\mathcal{L}_{*}=\cup_{n=0}^{\infty} \mathcal{L}_{n *}$. If $R \in \mathcal{L}_{n *}$, then $R=q_{n} / \omega_{n}$ with $q_{n} \in \Pi_{n}$ where

$$
\omega_{0}=1 \quad \text { and } \quad \omega_{n}(z)=\prod_{j=1}^{n}\left(z-\alpha_{j}\right) \quad \text { for } \quad n=1,2, \ldots
$$

Furthermore, let $\mathcal{R}=\mathcal{L}+\mathcal{L}_{*}$ and for $m$ and $n$ nonnegative integers, denote

$$
\mathcal{R}_{m, n}=\mathcal{L}_{m *}+\mathcal{L}_{n}=\left\{\frac{p}{\omega_{m} \pi_{n}}: p \in \Pi_{m+n}\right\} .
$$


Note that $B_{n *}=1 / B_{n}$ and hence $\mathcal{R}_{m, n}=\operatorname{span}\left\{1 / B_{m}, \ldots, 1 / B_{1}, 1, B_{1}, \ldots, B_{n}\right\}$ and $\mathcal{R}_{0, n}=$ $\mathcal{L}_{n}$. When all $\alpha_{k}=0$, then $\mathcal{R}_{m, n}=\Lambda_{m, n}$ and $\mathcal{L}_{n}=\Pi_{n}$.

We now suppose that the functional is defined on the space $\mathcal{R} \cdot \mathcal{R}$ and still has the same properties as before, then we have an inner product (1.1) defined on $\mathcal{R}$. Note that $\mathcal{R}_{*}=\mathcal{R}$, just like $\Lambda=\Lambda_{*}$, but unlike $\Lambda=\Lambda \cdot \Lambda=\Lambda+\Lambda$ we have $\mathcal{R} \neq \mathcal{R} \cdot \mathcal{R}=\mathcal{R}+\mathcal{R}$. Thus $M$ should be defined on $\mathcal{R} \cdot \mathcal{R}$ (and not just on $\mathcal{R}$ ) to have an inner product in $\mathcal{R}$.

The natural rational generalization of the orthogonal Laurent polynomials is then to orthogonalize the basis

$$
B_{0}, B_{1}, 1 / B_{1}, B_{2}, 1 / B_{2}, \ldots \quad \text { or } \quad B_{0}, 1 / B_{1}, B_{1}, 1 / B_{2}, B_{2}, \ldots
$$

In the first case, this gives rise to an orthogonal basis $\left\{\sigma_{n}\right\}$ for $\mathcal{R}$ and in the second case we find an orthogonal basis $\left\{\tau_{n}\right\}$. These orthogonal bases, their recurrences, interrelations and interpolating properties will be the subject of this paper.

\section{The orthogonal rational functions}

Our first concern is to show that these orthogonal rational functions $\sigma_{n}$ and $\tau_{n}$ can be obtained from analytic (in $\mathbb{D} \cup \mathbb{T}$ ) orthogonal rational functions associated with some auxiliary spaces $\tilde{\mathcal{L}}_{n}$ which are of the same type as the spaces $\mathcal{L}_{n}$ but now with respect to some auxiliary set of points $\tilde{\alpha}$. This is particularly interesting since it will allow us to use the now well developed machinery for these orthogonal functions.

We first discuss the sequence of orthogonal rational functions $\left\{\sigma_{n}\right\}_{0}^{\infty}$ which corresponds to the nesting

$$
\mathcal{R}_{0,0} \subset \mathcal{R}_{0,1} \subset \mathcal{R}_{1,1} \subset \mathcal{R}_{1,2} \subset \mathcal{R}_{2,2} \subset \cdots
$$

Using the notation

$$
\mathcal{R}_{2 n}=\mathcal{R}_{n, n} \quad \text { and } \quad \mathcal{R}_{2 n+1}=\mathcal{R}_{n, n+1} \quad \text { for } \quad n=0,1, \ldots
$$

we should have $\sigma_{n} \in \mathcal{R}_{n}-\mathcal{R}_{n-1}$ and $\sigma_{n} \perp \mathcal{R}_{n-1}$. We see that $\left\{\mathcal{R}_{n}\right\}_{0}^{\infty}$ represents a sequence of subspaces of $\mathcal{R}$ such that $\operatorname{dim}\left(\mathcal{R}_{n}\right)=n+1, \mathcal{R}_{n} \subset \mathcal{R}_{n+1}$, and $\mathcal{R}=\cup_{n=0}^{\infty} \mathcal{R}_{n}$.

Let us now consider the table of points $\left(\tilde{\alpha}_{0}=\alpha_{0}=0\right)$

$$
\tilde{\alpha}=\left\{\tilde{\alpha}_{0}, \tilde{\alpha}_{1}, \tilde{\alpha}_{2}, \tilde{\alpha}_{3}, \tilde{\alpha}_{4}, \ldots, \tilde{\alpha}_{2 m-1}, \tilde{\alpha}_{2 m}, \ldots\right\}=\left\{\alpha_{0}, \alpha_{1}, \alpha_{1}, \alpha_{2}, \alpha_{2}, \ldots, \alpha_{m}, \alpha_{m}, \ldots\right\} .
$$

Thus

$$
\tilde{\alpha}_{2 n}=\alpha_{n} \quad \text { and } \quad \tilde{\alpha}_{2 n+1}=\alpha_{n+1} \quad \text { for } \quad n=0,1, \ldots
$$

Let $\tilde{\zeta}_{n}(z)$ be the Blaschke factors for the table $\tilde{\alpha}$ and $\tilde{B}_{n}(z)$ the corresponding Blaschke products. Set $\tilde{\mathcal{L}}_{n}=\operatorname{span}\left\{1, \tilde{B}_{1}, \ldots, \tilde{B}_{n}\right\}$ and $\tilde{\mathcal{L}}=\cup_{n=0}^{\infty} \tilde{\mathcal{L}}_{n}$. Also, set $\tilde{\mathcal{L}}_{n *}=\operatorname{span}\left\{1, \tilde{B}_{1 *}, \ldots, \tilde{B}_{n *}\right\}$, $\tilde{\mathcal{L}}=\cup_{n=0}^{\infty} \tilde{\mathcal{L}}_{n *}$ and $\tilde{\mathcal{R}}_{m, n}=\tilde{\mathcal{L}}_{m *}+\tilde{\mathcal{L}}_{n}$.

One immediately sees that $\tilde{\mathcal{L}}=\mathcal{L} \cdot \mathcal{L}$ and since $\mathcal{R}=\mathcal{L} \cdot \mathcal{L}_{*}=\mathcal{R}_{*}$ and $\tilde{\mathcal{R}}=\tilde{\mathcal{L}} \cdot \tilde{\mathcal{L}}_{*}$, it also follows that $\tilde{\mathcal{R}}=\mathcal{L} \cdot \mathcal{L} \cdot \mathcal{L}_{*} \cdot \mathcal{L}_{*}=\mathcal{R} \cdot \mathcal{R}=\mathcal{R} \cdot \mathcal{R}_{*}$. Thus defining a linear functional $M$ on $\tilde{\mathcal{R}}=\mathcal{R} \cdot \mathcal{R}_{*}$ is the same as defining a linear functional on $\tilde{\mathcal{R}}=\tilde{\mathcal{L}} \cdot \tilde{\mathcal{L}}_{*}$ and we thus have an inner product on $\mathcal{R}$ as well as on $\tilde{\mathcal{L}}$.

Let $\left\{\tilde{\phi}_{n}\right\}_{n=0}^{\infty}$ be the orthogonal functions in $\tilde{\mathcal{L}}$ obtained by orthogonalizing the basis $\left\{1, \tilde{B}_{1}, \tilde{B}_{2}, \ldots\right\}$ corresponding to the table $\tilde{\alpha}$ in $(2.3)$. Note that by $(2.4)$

$$
\tilde{B}_{2 n}=B_{n}^{2} \quad \text { and } \quad \tilde{B}_{2 n+1}=B_{n+1} B_{n}, \quad n \geq 0 .
$$


Introducing the superstar conjugate as

$$
\tilde{\phi}_{n}^{*}(z)=\tilde{B}_{n}(z) \tilde{\phi}_{n *}(z) .
$$

and the functions

$$
\sigma_{2 n}(z)=B_{n *}(z) \tilde{\phi}_{2 n}^{*}(z) \text { and } \sigma_{2 n+1}(z)=B_{n *}(z) \tilde{\phi}_{2 n+1}(z),
$$

we have the following

Lemma 2.1 With $\sigma_{n}$ as in (2.6) and $\mathcal{R}_{n}$ as in (2.2), we have $\sigma_{n} \in \mathcal{R}_{n}$.

Proof. For $n$ even, we have to prove that $\sigma_{2 m} \in \mathcal{R}_{m, m}$. Because

$$
\sigma_{2 m}=B_{m *} \tilde{\phi}_{2 m}^{*}=\frac{\tilde{B}_{2 m} \tilde{\phi}_{2 m *}}{B_{m}}=B_{m} \tilde{\phi}_{2 m *}=\frac{p_{2 m}}{\omega_{m} \pi_{m}}, \quad p_{2 m} \in \Pi_{2 m}
$$

we have $\sigma_{2 m} \in \mathcal{L}_{m *} \cdot \mathcal{L}_{m}=\mathcal{R}_{m, m}$.

For $n$ odd, we have to show that $\sigma_{2 m+1} \in \mathcal{R}_{m, m+1}$. We have by a similar argument

$$
\sigma_{2 m+1}=B_{m *} \tilde{\phi}_{2 m+1}=\frac{r_{2 m+1}}{\pi_{m} \pi_{m+1} B_{m}}=\frac{q_{2 m+1}}{\pi_{m+1} \omega_{m}} \in \mathcal{L}_{m *} \cdot \mathcal{L}_{m+1}=\mathcal{R}_{m, m+1}
$$

where $r_{2 m+1}$ and $q_{2 m+1}$ are in $\Pi_{2 m+1}$.

This lemma says that the sequence $\left\{\sigma_{n}\right\}_{0}^{\infty}$ spans $\mathcal{R}$ according to the nesting (2.1), thus that $\left\{\sigma_{k}\right\}_{0}^{n}$ is a basis for $\mathcal{R}_{n}$ for all $n \geq 0$.

Theorem 2.2 The sequence $\left\{\sigma_{n}\right\}_{0}^{\infty}$ defined by (2.6) is an orthogonal basis for $\mathcal{R}$ with respect to the inner product induced by the linear functional $M$ and it respects the ordering (2.1). This means that $\mathcal{R}_{n-1, n} \perp \sigma_{2 n} \in \mathcal{R}_{n, n}$ and $\mathcal{R}_{n, n} \perp \sigma_{2 n+1} \in \mathcal{R}_{n, n+1}$ for $n=0,1, \ldots$

Proof. First note that for $f, g \in \tilde{\mathcal{L}}_{n}$ we have $\langle f, g\rangle=\left\langle g_{*}, f_{*}\right\rangle=\left\langle g^{*}, f^{*}\right\rangle$. Also $\tilde{\mathcal{L}}_{n}=\left\{g^{*}\right.$ : $\left.g \in \tilde{\mathcal{L}}_{n}\right\}$. Thus, since $\tilde{\phi}_{n} \perp \tilde{\mathcal{L}}_{n-1}$ we have

$$
0=\left\langle g, \tilde{\phi}_{n}\right\rangle=\left\langle\tilde{\phi}_{n}^{*}, \tilde{\zeta}_{n} g^{*}\right\rangle, \quad \forall g \in \tilde{\mathcal{L}}_{n-1}
$$

so that $\tilde{\phi}_{n}^{*} \perp \tilde{\zeta}_{n} \tilde{\mathcal{L}}_{n-1}$.

We first prove that $\sigma_{2 n} \perp \mathcal{R}_{n-1, n}=\mathcal{L}_{(n-1) *}+\mathcal{L}_{n}$ for $n \geq 1$. Therefore we prove that $\sigma_{2 n} \perp \mathcal{L}_{n}$ and that $\sigma_{2 n} \perp \mathcal{L}_{(n-1) *}$ separately.

Suppose that $f \in \mathcal{L}_{n}$, then $f=q_{n} / \pi_{n}$ with $q_{n} \in \Pi_{n}$. Then we have polynomials $p_{n}$ and $q_{n} \in \Pi_{n}$ such that

$$
B_{n-1} f=B_{n-1} \frac{q_{n}}{\pi_{n}}=\frac{\omega_{n-1} p_{n}}{\pi_{n-1} \pi_{n}} \in \tilde{\mathcal{L}}_{2 n-1}
$$

Thus

$$
\left\langle\sigma_{2 n}, f\right\rangle=\left\langle\tilde{\phi}_{2 n}^{*} B_{n *}, f\right\rangle=\left\langle\tilde{\phi}_{2 n}^{*}, B_{n} f\right\rangle=\left\langle\tilde{\phi}_{2 n}^{*}, \zeta_{n} B_{n-1} f\right\rangle=0, \quad \forall f \in \mathcal{L}_{n}
$$

because $\tilde{\phi}_{2 n}^{*} \perp \tilde{\zeta}_{2 n} \tilde{\mathcal{L}}_{2 n-1}=\zeta_{n} \tilde{\mathcal{L}}_{2 n-1}$. We may thus conclude that $\sigma_{2 n}$ is orthogonal to $\mathcal{L}_{n}$. Similarly we show that $\sigma_{2 n} \perp \mathcal{L}_{(n-1) *}$. Again we have

$$
\left\langle\sigma_{2 n}, f_{*}\right\rangle=\left\langle\tilde{\phi}_{2 n}^{*} B_{n *}, f_{*}\right\rangle=\left\langle\tilde{\phi}_{2 n}^{*}, \zeta_{n} f^{*}\right\rangle=0, \quad \forall f \in \mathcal{L}_{(n-1) *}
$$


because we can use the same orthogonality relation for $\tilde{\phi}_{2 n}^{*}$ since $f^{*} \in \mathcal{L}_{n-1} \subset \tilde{\mathcal{L}}_{2 n-1}$. Thus $\sigma_{2 n}$ is orthogonal to $\mathcal{L}_{n}$ and to $\mathcal{L}_{(n-1) *}$, hence orthogonal to $\mathcal{R}_{n-1, n}=\mathcal{L}_{(n-1) *}+\mathcal{L}_{n}$. $\mathcal{L}_{n *}$.

In the same way we prove that $\sigma_{2 n+1} \perp \mathcal{R}_{n, n}$ by showing that $\sigma_{2 n+1} \perp \mathcal{L}_{n}$ and $\sigma_{2 n+1} \perp$

Suppose $f \in \mathcal{L}_{n}$, then

$$
\left\langle\sigma_{2 n+1}, f\right\rangle=\left\langle\tilde{\phi}_{2 n+1} B_{n *}, f\right\rangle=\left\langle\tilde{\phi}_{2 n+1}, B_{n} f\right\rangle=0
$$

because $B_{n} f \in \tilde{\mathcal{L}}_{2 n} \perp \tilde{\phi}_{2 n+1}$. Thus $\sigma_{2 n+1} \perp \mathcal{L}_{n}$. On the other hand, for $f \in \mathcal{L}_{n}$

$$
\left\langle\sigma_{2 n+1}, f_{*}\right\rangle=\left\langle\tilde{\phi}_{2 n+1} B_{n *}, f_{*}\right\rangle=\left\langle\tilde{\phi}_{2 n+1}, B_{n} f_{*}\right\rangle=\left\langle\tilde{\phi}_{2 n+1}, f^{*}\right\rangle=0
$$

because $f^{*} \in \mathcal{L}_{n} \subset \tilde{\mathcal{L}}_{2 n} \perp \tilde{\phi}_{2 n+1}$. Thus $\sigma_{2 n+1} \perp \mathcal{L}_{n *}$. This proves the theorem.

For the ordering

$$
\mathcal{R}_{0,0} \subset \mathcal{R}_{1,0} \subset \mathcal{R}_{1,1} \subset \mathcal{R}_{2,1} \subset \cdots
$$

we can derive in a completely analogous way another orthogonal basis for $\mathcal{R}$ which turns out to be given by

$$
\tau_{2 n}=\tilde{\phi}_{2 n} B_{n *} \quad \text { and } \quad \tau_{2 n+1}=\tilde{\phi}_{2 n+1}^{*} B_{(n+1) *}
$$

and we have

Theorem 2.3 The sequence $\left\{\tau_{n}\right\}_{0}^{\infty}$ defined in (2.8) is an orthogonal basis for $\mathcal{R}$ with respect to the inner product induced by the linear functional $M$ and it respects the ordering (2.7). This means that $\mathcal{R}_{n, n-1} \perp \tau_{2 n} \in \mathcal{R}_{n, n}$ and $\mathcal{R}_{n, n} \perp \tau_{2 n+1} \in \mathcal{R}_{n+1, n}$ for $n=0,1, \ldots$

We remark that when all $\alpha_{k}=0$, then $\mathcal{L}=\Pi, \tilde{\mathcal{L}}=\mathcal{L} \cdot \mathcal{L}=\Pi, \mathcal{L}+\mathcal{L}_{*}=\mathcal{L} \cdot \mathcal{L}_{*}=\mathcal{R}=\Lambda$ and $\tilde{\mathcal{L}}+\tilde{\mathcal{L}}_{*}=\tilde{\mathcal{L}} \cdot \tilde{\mathcal{L}}_{*}=\Lambda$. Then the $\tilde{\phi}_{n}$ are the orthogonal (Szegö) polynomials and since $B_{n}(z)=z^{n}$

$$
\sigma_{2 n}(z)=z^{-n} \tilde{\phi}_{2 n}^{*}(z) \quad \text { and } \quad \sigma_{2 n+1}(z)=z^{-n} \tilde{\phi}_{2 n+1}(z)
$$

while

$$
\tau_{2 n}(z)=z^{-n} \tilde{\phi}_{2 n}(z) \quad \text { and } \quad \tau_{2 n+1}(z)=z^{-(n+1)} \tilde{\phi}_{2 n+1}^{*}(z) .
$$

These are the orthogonal Laurent polynomials given by Thron [16, Thm. 1].

\section{Recurrence relations and continued fractions}

Now that we have related the orthogonal bases $\sigma_{n}$ and $\tau_{n}$ with the orthogonal basis $\tilde{\phi}_{n}$, we can use the recurrence relations for the basis $\tilde{\phi}_{n}$ to obtain recurrence relations for the $\sigma_{n}$ and $\tau_{n}$.

We first recall the recurrence relations for the orthogonal functions for the spaces $\tilde{\mathcal{L}}_{n}$, associated with the table $\tilde{\alpha}$. Suppose we normalize the orthogonal functions to be "monic". This means that we set $\tilde{\Phi}_{n}^{*}\left(\tilde{\alpha}_{n}\right)=1$ or equivalently that in $\widetilde{\Phi}_{n}(z)=\sum_{k=0}^{n} a_{k, n} \tilde{B}_{k}(z)$ we set $a_{n, n}=1$. These monic rational functions are denoted with a capital greek letter.

The recurrence relations are given in [7, Thms. 3.1 and 3.4]:

$$
\tilde{\Phi}_{0}=\tilde{\Phi}_{0}^{*}=1
$$


and for $n=1,2, \ldots$

$$
\tilde{\Phi}_{n}^{*}(z)=-\tilde{z}_{n} \overline{\tilde{\theta}}_{n} \frac{z-\tilde{\alpha}_{n-1}}{1-\overline{\tilde{\alpha}}_{n} z} \tilde{\Phi}_{n-1}(z)-\tilde{z}_{n} \overline{\tilde{\nu}}_{n} \frac{1-\overline{\tilde{\alpha}}_{n-1} z}{1-\overline{\tilde{\alpha}}_{n} z} \tilde{\Phi}_{n-1}^{*}(z)
$$

and

$$
\tilde{\Phi}_{n}(z)=-\overline{\tilde{z}}_{n} \frac{\tilde{\theta}_{n}}{\overline{\tilde{\nu}}_{n}} \tilde{\Phi}_{n}^{*}(z)+\frac{1}{\overline{\tilde{\nu}}_{n}}\left[\left|\tilde{\nu}_{n}\right|^{2}-\left|\tilde{\theta}_{n}\right|^{2}\right] \frac{z-\tilde{\alpha}_{n-1}}{1-\tilde{\tilde{\alpha}}_{n} z} \tilde{\Phi}_{n-1}(z)
$$

with

$$
\begin{aligned}
& \tilde{\theta}_{n}=\frac{\left(1-\tilde{\alpha}_{n-1} \overline{\tilde{\alpha}}_{n}\right) \tilde{\Phi}_{n}\left(\tilde{\alpha}_{n-1}\right)}{1-\left|\tilde{\alpha}_{n-1}\right|^{2}} \\
& \tilde{\nu}_{n}=-\tilde{z}_{n} \frac{\left(1-\overline{\tilde{\alpha}}_{n-1} \tilde{\alpha}_{n}\right) \overline{\tilde{\Phi}_{n}^{*}\left(\tilde{\alpha}_{n-1}\right)}}{1-\left|\tilde{\alpha}_{n-1}\right|^{2}}
\end{aligned}
$$

and it holds that $\left|\tilde{\theta}_{n}\right|<\left|\tilde{\nu}_{n}\right|$. If $z_{n}$ is as in (1.3), then by (2.4) we have $\tilde{z}_{2 n}=z_{n}$ and $\tilde{z}_{2 n+1}=z_{n+1}$ for $n=0,1, \ldots$. Setting also $\delta_{n}=\tilde{\theta}_{n}$ and $\epsilon_{n}=\tilde{\nu}_{n}$ for $n \geq 1$, the recurrences can be written in the following somewhat simpler forms which hold for $n=0,1, \ldots$

$$
\begin{aligned}
& \tilde{\Phi}_{2 n+1}^{*}(z)=-z_{n+1} \bar{\delta}_{2 n+1} \frac{z-\alpha_{n}}{1-\bar{\alpha}_{n+1} z} \tilde{\Phi}_{2 n}(z)-z_{n+1} \bar{\epsilon}_{2 n+1} \frac{1-\bar{\alpha}_{n} z}{1-\bar{\alpha}_{n+1} z} \tilde{\Phi}_{2 n}^{*}(z) \\
& \tilde{\Phi}_{2 n+1}(z)=-\bar{z}_{n+1} \frac{\delta_{2 n+1}}{\bar{\epsilon}_{2 n+1}} \tilde{\Phi}_{2 n+1}^{*}(z)+\frac{1}{\bar{\epsilon}_{2 n+1}}\left[\left|\epsilon_{2 n+1}\right|^{2}-\left|\delta_{2 n+1}\right|^{2}\right] \frac{z-\alpha_{n}}{1-\bar{\alpha}_{n+1} z} \tilde{\Phi}_{2 n}(z) \\
& \tilde{\Phi}_{2 n+2}^{*}(z)=-z_{n+1} \bar{\delta}_{2 n+2} \frac{z-\alpha_{n+1}}{1-\bar{\alpha}_{n+1} z} \tilde{\Phi}_{2 n+1}(z)-z_{n+1} \bar{\epsilon}_{2 n+2} \tilde{\Phi}_{2 n+1}^{*}(z) \\
& \tilde{\Phi}_{2 n+2}(z)=-\bar{z}_{n+1} \frac{\delta_{2 n+2}}{\bar{\epsilon}_{2 n+2}} \tilde{\Phi}_{2 n+2}^{*}(z)+\frac{1}{\bar{\epsilon}_{2 n+2}}\left[\left|\epsilon_{2 n+2}\right|^{2}-\left|\delta_{2 n+2}\right|^{2}\right] \frac{1-\bar{\alpha}_{n+1} z}{1-\bar{\alpha}_{n+2} z} \tilde{\Phi}_{2 n+2}^{*}(z)
\end{aligned}
$$

with

$$
\begin{aligned}
\delta_{2 n} & =\tilde{\Phi}_{2 n}\left(\alpha_{n}\right), & \delta_{2 n+1} & =\frac{1-\alpha_{n} \bar{\alpha}_{n+1}}{1-\left|\alpha_{n}\right|^{2}} \tilde{\Phi}_{2 n+1}\left(\alpha_{n}\right) \\
\epsilon_{2 n} & =-z_{n} \overline{\tilde{\Phi}_{2 n}^{*}\left(\alpha_{n}\right)}, & \epsilon_{2 n+1} & =-z_{n+1} \frac{1-\bar{\alpha}_{n} \alpha_{n+1} \overline{\tilde{\Phi}_{2 n+1}^{*}\left(\alpha_{n}\right)}}{1-\left|\alpha_{n}\right|^{2}}
\end{aligned}
$$

Note that, although $\delta_{0}$ and $\epsilon_{0}$ do not appear in (3.1)-(3.4), they are defined by (3.5)-(3.6) as $\delta_{0}=\epsilon_{0}=1$. This will be used later. Suppose that for $n=0,1, \ldots$ we introduce the notation $(\kappa \in \mathbb{C}$ is arbitrary)

$$
\begin{array}{ll}
c_{1}=-2 \kappa, & d_{0}=\kappa, \quad d_{1}=1, \\
c_{4 n-2}=-z_{n} \bar{\epsilon}_{2 n-1} \frac{1-\bar{\alpha}_{n-1} z}{1-\bar{\alpha}_{n} z}, & d_{4 n-2}=-z_{n} \bar{\delta}_{2 n-1} \frac{z-\alpha_{n-1}}{1-\bar{\alpha}_{n} z} \\
c_{4 n-1}=\frac{1}{\bar{\epsilon}_{2 n-1}}\left[\left|\epsilon_{2 n-1}\right|^{2}-\left|\delta_{2 n-1}\right|^{2}\right] \frac{z-\alpha_{n-1}}{1-\bar{\alpha}_{n} z}, & d_{4 n-1}=-\bar{z}_{n} \frac{\delta_{2 n-1}}{\bar{\epsilon}_{2 n-1}} \\
c_{4 n}=-z_{n} \bar{\epsilon}_{2 n}, & d_{4 n}=-z_{n} \bar{\delta}_{2 n} \frac{z-\alpha_{n}}{1-\bar{\alpha}_{n} z} \\
c_{4 n+1}=\frac{1}{\bar{\epsilon}_{2 n}}\left[\left|\epsilon_{2 n}\right|^{2}-\left|\delta_{2 n}\right|^{2}\right] \frac{z-\alpha_{n}}{1-\bar{\alpha}_{n} z}, & d_{4 n+1}=-\bar{z}_{n} \frac{\delta_{2 n}}{\epsilon_{2 n}}
\end{array}
$$


and set $D_{-1}=0$ and for $n=0,1, \ldots$

$$
D_{4 n}=\tilde{\Phi}_{2 n}^{*}, \quad D_{4 n+1}=\tilde{\Phi}_{2 n}, \quad D_{4 n+2}=\tilde{\Phi}_{2 n+1}^{*}, \quad D_{4 n+3}=\tilde{\Phi}_{2 n+3}
$$

then the previous recurrences (3.1)-(3.6) reduce to

$$
D_{-1}=0, \quad D_{0}=1, \quad D_{n}=d_{n} D_{n-1}+c_{n} D_{n-2}, \quad n=1,2, \ldots
$$

In other words, the $D_{n}$ are the canonical denominators in the continued fraction $d_{0}+$ $\mathrm{K}_{n=1}^{\infty}\left(c_{n} / d_{n}\right)$ where $c_{n}$ and $d_{n}$ are given by (3.7)-(3.11). So we can denote its approximants as $C_{n} / D_{n}, n \geq 0$. Note that when all $\alpha_{n}=0$, this is a PC fraction (Perron-Carathéodory continued fraction) [16].

Now suppose that the orthogonal functions $\sigma_{n}$ and $\tau_{n}$ are normalized such that we have chosen in (2.6) and (2.8) the monic functions $\tilde{\Phi}_{n}$. Thus

$$
\begin{array}{lll}
\sigma_{2 n}=B_{n *} \tilde{\Phi}_{2 n}^{*}, & \sigma_{2 n+1}=B_{n *} \tilde{\Phi}_{2 n+1}, & n=0,1, \ldots \\
\tau_{2 n}=B_{n *} \tilde{\Phi}_{2 n}, & \tau_{2 n+1}=B_{(n+1) *} \tilde{\Phi}_{2 n+1}^{*}, & n=0,1, \ldots
\end{array}
$$

We recall that $\tilde{B}_{n}$ is given by $(2.5)$ so that

$$
\tilde{\Phi}_{2 n}^{*}=\tilde{\Phi}_{2 n *} B_{n} B_{n} \quad \text { and } \quad \tilde{\Phi}_{2 n+1}^{*}=\tilde{\Phi}_{(2 n+1) *} B_{n} B_{n+1}, \quad n=0,1, \ldots
$$

We now want to construct a continued fraction $f_{0}+\mathrm{K}_{n=1}^{\infty}\left(e_{n} / f_{n}\right)$ which is equivalent to $d_{0}+\mathrm{K}_{n=1}^{\infty}\left(c_{n} / d_{n}\right)$ and whose canonical numerators and denominators are $E_{n}$ and $F_{n}$ such that the denominators are given by

$$
F_{4 n}=\sigma_{2 n}, \quad F_{4 n+1}=\tau_{2 n}, \quad F_{4 n+2}=\tau_{2 n+1}, \quad F_{4 n+3}=\sigma_{2 n+1}, \quad n=0,1, \ldots
$$

By the formulas for equivalence transformations given in $[9,1,13]$, or directly working on the recurrence relations (compare with [16] for the polynomial case), it is seen that we then need a sequence $\left\{\rho_{n}: n=0,1,2, \ldots\right\}$ with $\rho_{0}=1$, giving an equivalent continued fraction with

$$
e_{n}=\rho_{n} \rho_{n-1} c_{n} \quad \text { for } n \geq 1 \text { and } \quad f_{n}=\rho_{n} d_{n} \text { for } n \geq 0 .
$$

Thus to get the desired result, these $\rho$ 's should satisfy

$$
\begin{aligned}
B_{n *} \tilde{\Phi}_{2 n}^{*} & =\rho_{0} \cdots \rho_{4 n} \tilde{\Phi}_{2 n}^{*} \\
B_{n *} \tilde{\Phi}_{2 n} & =\rho_{0} \cdots \rho_{4 n+1} \tilde{\Phi}_{2 n} \\
B_{(n+1) *} \tilde{\Phi}_{2 n+1}^{*} & =\rho_{0} \cdots \rho_{4 n+2} \tilde{\Phi}_{2 n+1}^{*} \\
B_{n *} \tilde{\Phi}_{2 n+1} & =\rho_{0} \cdots \rho_{4 n+3} \tilde{\Phi}_{2 n+1} .
\end{aligned}
$$

From (3.17)-(3.20) it then follows that for $n=1,2, \ldots$

$$
\begin{array}{ll}
\rho_{4 n-3}=1, & \rho_{4 n-2}=\frac{1-\bar{\alpha}_{n} z}{z_{n}\left(\alpha_{n}-z\right)}, \\
\rho_{4 n-1}=\frac{z_{n}\left(\alpha_{n}-z\right)}{1-\bar{\alpha}_{n} z}, & \rho_{4 n}=\frac{1-\bar{\alpha}_{n} z}{z_{n}\left(\alpha_{n}-z\right)} .
\end{array}
$$

We then conclude from (3.7)-(3.11), (3.16) and (3.21)-(3.22) that

$$
e_{1}=-2 \kappa, \quad f_{0}=\kappa, \quad f_{1}=1,
$$


and for $n=1,2, \ldots$

$$
\begin{array}{ll}
e_{4 n-2}=\bar{\epsilon}_{2 n-1} \frac{1-\bar{\alpha}_{n-1} z}{z-\alpha_{n}}, & f_{4 n-2}=\bar{\delta}_{2 n-1} \frac{z-\alpha_{n-1}}{z-\alpha_{n}} \\
e_{4 n-1}=\frac{1}{\bar{\epsilon}_{2 n-1}}\left[\left|\epsilon_{2 n-1}\right|^{2}-\left|\delta_{2 n-1}\right|^{2}\right] \frac{z-\alpha_{n-1}}{1-\bar{\alpha}_{n} z}, & f_{4 n-1}=\frac{\delta_{2 n-1}}{\bar{\epsilon}_{2 n-1}} \frac{\left(z-\alpha_{n}\right)}{\left(1-\bar{\alpha}_{n} z\right)} \\
e_{4 n}=-z_{n} \bar{\epsilon}_{2 n}, & f_{4 n}=\bar{\delta}_{2 n} \\
e_{4 n+1}=-\frac{\bar{z}_{n}}{\epsilon_{2 n}}\left[\left|\epsilon_{2 n}\right|^{2}-\left|\delta_{2 n}\right|^{2}\right], & f_{4 n+1}=-\bar{z}_{n} \frac{\delta_{2 n}}{\epsilon_{2 n}}
\end{array}
$$

The approximants $E_{n} / F_{n}$ of the continued fraction $f_{0}+\mathrm{K}_{n=0}^{\infty}\left(e_{n} / f_{n}\right)$ are equal to the approximants $C_{n} / D_{n}$ and the canonical denominators $F_{n}$ satisfy (3.15).

Thus we have proved

Lemma 3.1 The continued fraction $f_{0}+\mathrm{K}_{n=1}^{\infty}\left(e_{n} / f_{n}\right)$ with $\left(e_{n}, f_{n}\right)$ as given by (3.23)-(3.27) is equivalent with the continued fraction $d_{0}+\mathrm{K}_{k=1}^{\infty}\left(c_{n} / d_{n}\right)$ with $\left(c_{n}, d_{n}\right)$ given by (3.7)-(3.11) and its canonical denominators are the $F_{n}$ given by (3.15) where the $\sigma_{n}$ and $\tau_{n}$ are the orthogonal rational functions for $\mathcal{R}$ which are given by (3.12)-(3.13).

This gives us explicit formulas for recurrence relations satisfied by the orthogonal rational functions $\sigma_{n}$ and $\tau_{n}$. We just have to write down the recurrence for the denominators $F_{n}$. These are mixed relations in the sense that the recurrence for the $\sigma$ 's and the $\tau$ 's is intertwined. We give them explicitly.

Theorem 3.2 The orthogonal rational functions $\sigma_{n}$ and $\tau_{n}$ for $\mathcal{R}$ given by (3.12)-(3.13) satisfy the mixed recurrence relations

$$
\sigma_{0}=\tau_{0}=1
$$

and for $n=1,2, \ldots$

$$
\begin{aligned}
\tau_{2 n-1} & =\bar{\delta}_{2 n-1} \frac{z-\alpha_{n-1}}{a-\alpha_{n}} \tau_{2 n-2}-\bar{\epsilon}_{2 n-1} \frac{1-\bar{\alpha}_{n-1} z}{z-\alpha_{n}} \sigma_{2 n-2} \\
\sigma_{2 n-1} & =\frac{\delta_{2 n-1}}{\bar{\epsilon}_{2 n-1}} \frac{z-\alpha_{n}}{1-\bar{\alpha}_{n} z} \tau_{2 n-1}+\frac{1}{\bar{\epsilon}_{2 n-1}}\left[\left|\epsilon_{2 n-1}\right|^{2}-\left|\delta_{2 n-1}\right|^{2}\right] \frac{z-\alpha_{n-1}}{1-\bar{\alpha}_{n} z} \tau_{2 n-2} \\
\sigma_{2 n} & =\bar{\delta}_{2 n} \sigma_{2 n-1}-z_{n} \bar{\epsilon}_{2 n} \tau_{2 n-1} \\
\tau_{2 n} & =-\bar{z}_{n} \frac{\delta_{2 n}}{\epsilon_{2 n}} \sigma_{2 n}-\frac{\bar{z}_{n}}{\epsilon_{2 n}}\left[\left|\epsilon_{2 n}\right|^{2}-\left|\delta_{2 n}\right|^{2}\right] \tau_{2 n-1}, .
\end{aligned}
$$

where $\delta_{n}$ and $\epsilon_{n}$ are given by (3.5)-(3.6).

We now try to decouple these relations and get recurrences of the form

$$
\begin{aligned}
\sigma_{2 n} & =h_{2 n} \sigma_{2 n-1}+g_{2 n} \sigma_{2 n-2} \\
\sigma_{2 n+1} & =h_{2 n+1} \sigma_{2 n}+g_{2 n+1} \sigma_{2 n-1}
\end{aligned}
$$

Thus we try to find a continued fraction $h_{0}+\mathrm{K}_{n=1}^{\infty}\left(g_{n} / h_{n}\right)$ whose canonical denominators are $\sigma_{n}$. Therefore we should construct a contraction of $f_{0}+K_{n=1}^{\infty}\left(e_{n} / f_{n}\right)$ to $h_{0}+K_{n=1}^{\infty}\left(g_{n} / h_{n}\right)$ such 
that the canonical denominators are related by $F_{4 n}=H_{2 n}$ and $F_{4 n+3}=H_{2 n+1}$. In Thron's paper [16], such formulas can be found. The result is that $h_{0}=f_{0}$ and for $n=1,2, \ldots$

$$
\begin{aligned}
g_{2 n} & =\frac{e_{4 n} e_{4 n-1} e_{4 n-2}}{f_{4 n-1} f_{4 n-2}+e_{4 n-1}} \\
h_{2 n} & =f_{4 n}+\frac{e_{4 n} f_{4 n-2}}{f_{4 n-1} f_{4 n-2}+e_{4 n-1}} \\
g_{2 n-1} & =\left(f_{4 n-1} f_{4 n-2}+e_{4 n-1}\right) e_{4 n-3} \\
h_{2 n-1} & =\left(f_{4 n-1} f_{4 n-2} f_{4 n-3}+f_{4 n-1} e_{4 n-2}+e_{4 n-1} f_{4 n-3}\right) .
\end{aligned}
$$

Substituting from (3.24)-(3.27) into (3.35)-(3.38) we get for $n=1,2, \ldots$

$$
\begin{aligned}
g_{2 n} & =-z_{n} \frac{\bar{\epsilon}_{2 n}}{\epsilon_{2 n-1}}\left[\left|\epsilon_{2 n-1}\right|^{2}-\left|\delta_{2 n-1}\right|^{2}\right] \frac{1-\bar{\alpha}_{n-1} z}{z-\alpha_{n}} \\
h_{2 n} & =\bar{\delta}_{2 n}-z_{n} \frac{\bar{\epsilon}_{n}}{\epsilon_{2 n-1}} \bar{\delta}_{2 n-1} \frac{1-\bar{\alpha}_{n} z}{z-\alpha_{n}} \\
g_{2 n-1} & =-\frac{z_{n-1}}{\epsilon_{2 n-2} \bar{\epsilon}_{2 n-1}}\left[\left|\epsilon_{2 n-2}\right|^{2}-\left|\delta_{2 n-2}\right|^{2}\right] \frac{z-\alpha_{n-1}}{1-\bar{\alpha}_{n-1} z} \\
h_{2 n-1} & =-z_{n} \epsilon_{2 n-1} \frac{\delta_{2 n-2}}{\epsilon_{2 n-2}} \frac{z-\alpha_{n-1}}{1-\bar{\alpha}_{n} z}+\delta_{2 n-1} \frac{1-\bar{\alpha}_{n-1} z}{1-\bar{\alpha}_{n} z} .
\end{aligned}
$$

So we get the explicit recurrence formulas for $n=1,2,3, \ldots$

$$
\begin{aligned}
\sigma_{2 n}= & {\left[\bar{\delta}_{2 n}-z_{n} \frac{\bar{\epsilon}_{2 n}}{\epsilon_{2 n-1}} \bar{\delta}_{2 n-1} \frac{1-\bar{\alpha}_{n} z}{z-\alpha_{n}}\right] \sigma_{2 n-1} } \\
& -\left[z_{n} \frac{\bar{\epsilon}_{2 n}}{\epsilon_{2 n-1}}\left[\left|\epsilon_{2 n-1}\right|^{2}-\left|\delta_{2 n-1}\right|^{2}\right] \frac{1-\bar{\alpha}_{n-1} z}{z-\alpha_{n}}\right] \sigma_{2 n-2}, \\
\sigma_{2 n+1}= & {\left[\delta_{2 n+1} \frac{1-\bar{\alpha}_{n} z}{1-\bar{\alpha}_{n+1} z}-z_{n} \epsilon_{2 n+1} \frac{\delta_{2 n}}{\epsilon_{2 n}} \frac{z-\alpha_{n}}{1-\bar{\alpha}_{n+1} z}\right] \sigma_{2 n} } \\
& -\left[\frac{z_{n}}{\epsilon_{2 n} \bar{\epsilon}_{2 n+1}}\left[\left|\epsilon_{2 n}\right|^{2}-\left|\delta_{2 n}\right|^{2}\right] \frac{z-\alpha_{n}}{1-\bar{\alpha}_{n} z}\right] \sigma_{2 n-1},
\end{aligned}
$$

with initial conditions

$$
\sigma_{0}=1, \quad \sigma_{1}(z)=\frac{\delta_{1}+\epsilon_{1} z}{1-\bar{\alpha}_{1} z} .
$$

It is possible to give a similar derivation to obtain by another contraction the explicit recurrence relation for the $\tau_{n}$. However, from (3.12)-(3.14) it immediately follows that

$$
\sigma_{n *}=\tau_{n} .
$$

Thus, the recurrences for the $\tau_{n}$ can be obtained from the recurrences for the $\sigma_{n}$ by taking the substar conjugate. We get for $n=1,2, \ldots$

$$
\begin{aligned}
\tau_{2 n}= & {\left[\delta_{2 n}-\bar{z}_{n} \frac{\epsilon_{2 n}}{\bar{\epsilon}_{2 n-1}} \delta_{2 n-1} \frac{z-\alpha_{n}}{1-\bar{\alpha}_{n} z}\right] \tau_{2 n-1} } \\
& -\left[\bar{z}_{n} \frac{\epsilon_{2 n}}{\bar{\epsilon}_{2 n-1}}\left[\left|\epsilon_{2 n-1}\right|^{2}-\left|\delta_{2 n-1}\right|^{2}\right] \frac{z-\alpha_{n-1}}{1-\bar{\alpha}_{n} z}\right] \tau_{2 n-2}, \\
\tau_{2 n+1}= & {\left[\bar{\delta}_{2 n+1} \frac{z-\alpha_{n}}{z-\alpha_{n+1}}-\bar{z}_{n} \bar{\epsilon}_{2 n+1} \frac{\bar{\delta}_{2 n}}{\bar{\epsilon}_{2 n}} \frac{1-\bar{\alpha}_{n} z}{z-\alpha_{n+1}}\right] \tau_{2 n} } \\
& -\left[\frac{\bar{z}_{n}}{\bar{\epsilon}_{2 n} \epsilon_{2 n+1}}\left[\left|\epsilon_{2 n}\right|^{2}-\left|\delta_{2 n}\right|^{2}\right] \frac{1-\bar{\alpha}_{n} z}{z-\alpha_{n}}\right] \tau_{2 n-1} .
\end{aligned}
$$


However it is easily checked that by setting $\sigma_{-1}=\tau_{-1}=0$ and $\sigma_{0}=\tau_{0}=1$, the previous recurrences hold for $n=0,1, \ldots$. Thus we have

Theorem 3.3 The orthogonal rational functions $\sigma_{n}$ and $\tau_{n}$ for $\mathcal{R}$ given by (3.12)-(3.13) satisfy the three term recurrence relations (3.43)-(3.44) and (3.46)-(3.47) for $n=0,1,2, \ldots$, provided we set $\sigma_{-1}=\tau_{-1}=0$ and $\sigma_{0}=\tau_{0}=1$. The $\delta_{n}$ and $\epsilon_{n}$ are given by (3.5)-(3.6).

\section{Interpolation}

The continued fraction $f_{0}+\mathrm{K}_{n=1}^{\infty}\left(e_{n} / f_{n}\right)$ with $\left(e_{n}, f_{n}\right)$ given by (3.23)-(3.27) is completely defined by the linear functional $M$, the points $\left\{\alpha_{k}\right\}_{0}^{\infty}$ and the value of $f_{0}=\kappa$. We shall show that this continued fraction defines uniquely two formal Newton series $\Xi$ and $\Gamma$.

We recall that a formal Newton series with respect to the points $\beta=\left\{\beta_{k}: k=0,1, \ldots\right\}$ is a formal series of the form

$$
\nu_{0}+\nu_{1}\left(z-\beta_{0}\right)+\nu_{2}\left(z-\beta_{0}\right)\left(z-\beta_{1}\right)+\cdots+\nu_{n}\left(z-\beta_{0}\right) \cdots\left(z-\beta_{n-1}\right)+\cdots
$$

If $S_{n}$ denotes the polynomial given by sum of the first $n+1$ terms in this series, then the (formal) Newton series expansion of a function $\varphi$ with respect to $\beta$ is the unique Newton series of the form (4.1) which satisfies $\varphi(z)-S_{n}(z)=O\left[\left(z-\beta_{0}\right) \cdots\left(z-\beta_{n}\right)\right]$ for $n=$ $0,1,2, \ldots$ The coefficients $\left(\nu_{0}, \nu_{1}, \ldots\right)$ are the coordinates of $\varphi$ with respect to the Newton basis $\left\{N_{0}, N_{1}, N_{2}, \ldots\right\}$ where $N_{0}=1$ and $N_{n}=\prod_{k=1}^{n}\left(z-\beta_{k-1}\right)$ for $n \geq 1$, which can be associated with the interpolation points $\beta$. These coordinates are given by the (possibly confluent) divided differences $\nu_{k}=\varphi\left[\beta_{0}, \beta_{1}, \ldots, \beta_{k}\right]$.

If we assume $\alpha_{n} \neq 0$ for $n=1,2, \ldots$ and set $\hat{\alpha}_{n}=1 / \bar{\alpha}_{n}$, then we shall consider formal Newton series with repect to the tables

$$
\left\{0, \alpha_{1}, \hat{\alpha}_{1}, \alpha_{1}, \hat{\alpha}_{1}, \alpha_{2}, \hat{\alpha}_{2}, \alpha_{2}, \hat{\alpha}_{2}, \ldots, \alpha_{n}, \hat{\alpha}_{n}, \alpha_{n}, \hat{\alpha}_{n}, \ldots\right\}
$$

and

$$
\left\{0, \hat{\alpha}_{1}, \alpha_{1}, \hat{\alpha}_{1}, \alpha_{1}, \hat{\alpha}_{2}, \alpha_{2}, \hat{\alpha}_{2}, \alpha_{2}, \ldots, \hat{\alpha}_{n}, \alpha_{n}, \hat{\alpha}_{n}, \alpha_{n}, \ldots\right\}
$$

Obviously, here we can use the alternative Newton bases

$$
1, z, z \omega_{1}, z \omega_{1} \pi_{1}, z \omega_{1}^{2} \pi_{1}, z \omega_{1}^{2} \pi_{1}^{2}, \ldots, z \omega_{n}^{2} \pi_{n-1} \pi_{n}, z \omega_{n}^{2} \pi_{n}^{2}, z \omega_{n} \omega_{n+1} \pi_{n}^{2}, z \omega_{n} \omega_{n+1} \pi_{n} \pi_{n+1}, \ldots
$$
i.e.,

$$
\begin{aligned}
& V_{0}=1, \\
& V_{4 n+1}=z \omega_{n}^{2} \pi_{n}^{2}, \quad V_{4 n+2}=z \omega_{n} \omega_{n+1} \pi_{n}^{2}, \quad n=0,1,2, \ldots \\
& V_{4 n+3}=z \omega_{n} \omega_{n+1} \pi_{n} \pi_{n+1}, \quad V_{4 n+4}=z \omega_{n+1}^{2} \pi_{n} \pi_{n+1}, \quad n=0,1,2, \ldots
\end{aligned}
$$

for the first case and

$$
1, z, z \pi_{1}, z \omega_{1} \pi_{1}, z \omega_{1} \pi_{1}^{2}, z \omega_{1}^{2} \pi_{1}^{2}, \ldots, z \omega_{n-1} \omega_{n} \pi_{n}^{2}, z \omega_{n}^{2} \pi_{n}^{2}, z \omega_{n}^{2} \pi_{n} \pi_{n+1}, z \omega_{n} \omega_{n+1} \pi_{n} \pi_{n+1}, \ldots
$$

i.e.,

$$
\begin{aligned}
& W_{0}=1, \\
& W_{4 n+1}=z \omega_{n}^{2} \pi_{n}^{2}, \quad W_{4 n+2}=z \omega_{n}^{2} \pi_{n} \pi_{n+1}, \quad n=0,1,2, \ldots \\
& W_{4 n+3}=z \omega_{n} \omega_{n+1} \pi_{n} \pi_{n+1}, \quad W_{4 n+4}=z \omega_{n} \omega_{n+1} \pi_{n+1}^{2}, \quad n=0,1,2, \ldots
\end{aligned}
$$


in the second case

It is easily shown by an induction argument that the canonical numerators $E_{n}$ and denominators $F_{n}$ of the continued fraction $f_{0}+\mathrm{K}_{n=1}^{\infty}\left(e_{n} / f_{n}\right)$ are of the following form $(n \geq 0)$

$$
\begin{aligned}
& E_{4 n}=\frac{P_{4 n}}{\omega_{n} \pi_{n}}, \quad F_{4 n}=\frac{Q_{4 n}}{\omega_{n} \pi_{n}}, \quad P_{4 n}=\tilde{p}_{2 n}, \quad Q_{4 n}=p_{2 n}, \\
& E_{4 n+1}=\frac{P_{4 n+1}}{\omega_{n} \pi_{n}}, \quad F_{4 n+1}=\frac{Q_{4 n+1}}{\omega_{n} \pi_{n}}, \quad P_{4 n+1}=\tilde{q}_{2 n}, \quad Q_{4 n+1}=q_{2 n}, \\
& E_{4 n+2}=\frac{P_{4 n+2}}{\omega_{n+1} \pi_{n}}, \quad F_{4 n+2}=\frac{Q_{4 n+2}}{\omega_{n+1} \pi_{n}}, \quad P_{4 n+2}=\tilde{p}_{2 n+1}, \quad Q_{4 n+2}=p_{2 n+1}, \\
& E_{4 n+3}=\frac{P_{4 n+3}}{\omega_{n} \pi_{n+1}}, \quad F_{4 n+3}=\frac{Q_{4 n+3}}{\omega_{n} \pi_{n+1}}, \quad P_{4 n+3}=\tilde{q}_{2 n+1}, \quad Q_{4 n+3}=q_{2 n+1},
\end{aligned}
$$

where $\tilde{p}_{n}, p_{n}, \tilde{q}_{n}$, and $q_{n}$ are all in $\Pi_{n}$. Since $E_{n}$ and $F_{n}$ have the same denominator, we can define

$$
K_{n}=\frac{E_{n}}{F_{n}}=\frac{P_{n}}{Q_{n}}, \quad n=0,1,2, \ldots
$$

In the remainder of this section, we assume that the above expressions for $F_{n}$ are irreducible and do not vanish at the origin nor at infinity. We recall from [7] that the $n$th orthogonal rational function $\tilde{\Phi}_{n}$ for a sequence $\tilde{\alpha}$ is called non-degenerate if the numerator of $\tilde{\Phi}_{n}^{*}$ does not vanish in $\tilde{\alpha}_{n-1}$ and it is called non-exceptional if the numerator of $\tilde{\Phi}_{n}$ does not vanish in $\tilde{\alpha}_{n-1}$. We shall say that $\tilde{\Phi}_{n}$ is completely non-degenerate if the numerator of $\tilde{\Phi}_{n}^{*}$ does not vanish in any of the $\tilde{\alpha}_{k}, k=0,1, \ldots, n$ (for $k=n$ this is automatic, since otherwise $\tilde{\Phi}_{n}$ would not be in $\mathcal{R}_{n, n}$ ) and we call $\tilde{\Phi}_{n}$ completely non-exceptional if its numerator does not vanish in any of the $\alpha_{k}, k=0,1, \ldots, n$. If $\tilde{\Phi}_{n}$ is completely non-degenerate and completely non-exceptional, we shall call it completely regular. In [7, Proposition 2.1], it is proved that if the linear functional $M$ is positive definite and all $\tilde{\alpha}_{k}$ are in $\mathbb{D}$, then all of the $\tilde{\Phi}_{n}$ are non-degenerate, but the same proof also implies that the $\tilde{\Phi}_{n}$ are competely non-degenerate. Also recall (see the proof of Lemma 2.1) that the polynomials $p_{n}$ are numerators of some $\tilde{\phi}_{n}^{*}$ where the $\tilde{\phi}_{n}$ are orthogonal rational functions for the sequence $\tilde{\alpha} \subset \mathbb{D}$ and thus $p_{2 n}$ can not vanish in any of the points $0, \alpha_{1}, \ldots, \alpha_{n}$ while $p_{2 n+1}$ can not vanish in any of the points $0, \alpha_{1}, \ldots, \alpha_{n+1}$. Thus the above requirement for $F_{4 n}$ and $F_{4 n+2}$ comes down to saying that neither $p_{2 n}$ nor $p_{2 n+1}$ vanish in the points $\hat{\alpha}_{1}, \ldots, \hat{\alpha}_{n}$ and that they have precise degree as indicated by their indices. Similarly, since the $q_{n}$ appear as numerators of some orthogonal rational functions $\tilde{\phi}_{n}$, the above assumptions on $F_{4 n+1}$ and $F_{4 n+3}$ require that neither $q_{2 n}$ nor $q_{2 n+1}$ vanish in any of the points $0, \alpha_{1}, \ldots, \alpha_{n}$. These conditions mean that all the $\widetilde{\Phi}_{n}$ are completely regular.

We shall use the following general formulas for continued fractions

$$
E_{n} F_{n-1}-E_{n-1} F_{n}=(-1)^{n-1} e_{1} e_{2} \cdots e_{n}, \quad n=1,2, \ldots
$$

and

$$
E_{n+1} F_{n-1}-E_{n-1} F_{n+1}=(-1)^{n-1} e_{1} e_{2} \cdots e_{n} f_{n+1}, \quad n=1,2, \ldots
$$

where in our case, the expressions (3.23)-(3.27) give (the $w_{n}$ are constants)

$$
\begin{aligned}
& e_{1} e_{2} \cdots e_{4 n}=\frac{w_{4 n} z}{\left(z-\alpha_{n}\right)\left(1-\bar{\alpha}_{n} z\right)}, \quad n=1,2, \ldots \\
& e_{1} e_{2} \cdots e_{4 n+1}=\frac{w_{4 n+1} z}{\left(z-\alpha_{n}\right)\left(1-\bar{\alpha}_{n} z\right)}, \quad n=0,1, \ldots
\end{aligned}
$$




$$
\begin{aligned}
& e_{1} e_{2} \cdots e_{4 n+2}=\frac{w_{4 n+2} z}{\left(z-\alpha_{n}\right)\left(z-\alpha_{n+1}\right)}, \quad n=0,1, \ldots \\
& e_{1} e_{2} \cdots e_{4 n+3}=\frac{w_{4 n+3} z}{\left(z-\alpha_{n+1}\right)\left(1-\bar{\alpha}_{n+1} z\right)}, \quad n=0,1, \ldots
\end{aligned}
$$

When we set by convention $\omega_{-1}=z^{-1}$ and $\pi_{-1}=1$, then we see by direct verification for the initial conditions and by substituting (4.17)-(4.20) and (4.10)-(4.13) into (4.15)-(4.16), for the general cases that the following relations hold ( $u_{n}$ and $v_{n}$ are constants)

$$
\begin{aligned}
K_{4 n+1}-K_{4 n} & =u_{4 n} \frac{z \omega_{n-1} \omega_{n} \pi_{n-1} \pi_{n}}{p_{2 n} q_{2 n}}, \quad n=0,1, \ldots \\
K_{4 n+2}-K_{4 n+1} & =u_{4 n+1} \frac{z \omega_{n-1} \omega_{n} \pi_{n} \pi_{n}}{p_{2 n+1} q_{2 n}}, \quad n=0,1, \ldots \\
K_{4 n+3}-K_{4 n+2} & =u_{4 n+2} \frac{z \omega_{n} \omega_{n} \pi_{n} \pi_{n}}{p_{2 n+1} q_{2 n+1}}, \quad n=0,1, \ldots \\
K_{4 n+4}-K_{4 n+3} & =u_{4 n+3} \frac{z \omega_{n} \omega_{n} \pi_{n} \pi_{n+1}}{p_{2 n+2} q_{2 n+1}}, \quad n=0,1, \ldots \\
K_{4 n+1}-K_{4 n-1} & =v_{4 n-1} \frac{z \omega_{n-1} \omega_{n-1} \pi_{n-1} \pi_{n}}{q_{2 n} q_{2 n-1}}, \quad n=1,2, \ldots \\
K_{4 n+2}-K_{4 n} & =v_{4 n} \frac{z \omega_{n} \omega_{n} \pi_{n-1} \pi_{n}}{p_{2 n+1} p_{2 n}}, \quad n=0,1, \ldots \\
K_{4 n+3}-K_{4 n+1} & =v_{4 n+1} \frac{z \omega_{n-1} \omega_{n} \pi_{n} \pi_{n}}{q_{2 n+1} q_{2 n}}, \quad n=0,1, \ldots \\
K_{4 n+4}-K_{4 n+2} & =v_{4 n+2} \frac{z \omega_{n} \omega_{n+1} \pi_{n} \pi_{n}}{p_{2 n+1} p_{2 n+2}}, \quad n=0,1, \ldots
\end{aligned}
$$

Let us denote the coordinates of $K_{n}$ with respect to Newton basis (4.4)-(4.6) as $\left(\xi_{k}^{(n)}\right)_{k=0}^{\infty}$ and those with respect to the Newton basis $(4.7)-(4.9)$ by $\left(\gamma_{k}^{(n)}\right)_{k=0}^{\infty}$. Then, considering the even approximants, it follows from (4.26) and (4.28) that for $n \geq 1$

$$
\xi_{k}^{(4 n-2)}=\xi_{k}^{(4 n)}, \quad k=0,1, \ldots, 4 n-3, \quad \text { and } \quad \xi_{k}^{(4 n)}=\xi_{k}^{(4 n+2)}, \quad k=0,1, \ldots, 4 n-1 .
$$

Thus $\xi_{k}^{(2 n)}$ is independent of $n$ for $k \leq 2 n-1$, so that the numbers

$$
\xi_{k}=\xi_{k}^{(2 n)}, \quad k \leq 2 n-1
$$

for $k=0,1, \ldots$ are well defined and not depending on $n$.

Similarly it follows from (4.25) and (4.27) that for the odd approximants $(n \geq 1)$

$$
\gamma_{k}^{(4 n-1)}=\gamma_{k}^{(4 n+1)}, \quad k=0, \ldots, 4 n-3 \quad \text { and } \quad \gamma_{k}^{(4 n+1)}=\gamma_{k}^{(4 n+3)}, \quad k=0, \ldots, 4 n-1 .
$$

and the numbers

$$
\gamma_{k}=\gamma_{k}^{(2 n+1)}, \quad k \leq 2 n-1
$$

are defined independent from $n$ for $k=0,1,2, \ldots$

With these $\xi_{k}$ and $\gamma_{k}$, we can define the formal Newton series

$$
\Xi(z)=\xi_{0}+\xi_{1} V_{1}(z)+\xi_{2} V_{2}(z)+\cdots
$$

and

$$
\Gamma(z)=\gamma_{0}+\gamma_{1} W_{1}(z)+\gamma_{2} W_{2}(z)+\cdots
$$


with the Newton bases $V_{k}$ as in (4.4)-(4.6) and $W_{k}$ as in (4.7)-(4.9). Clearly $\xi_{0}=\gamma_{0}=\kappa$, with $f_{0}=\kappa$ the common value at $z=0$ for all $K_{n}$, with the exception of $n=1$ (where the value is $-\kappa)$. Thus we have

$$
K_{n}(0)=\xi_{0}=\gamma_{0}=\kappa, \quad n=0,2,3,4, \ldots
$$

On the other hand, by (4.21)-(4.24), we see that for $n=2,3, \ldots$

$$
K_{n+1}-K_{n}=O\left(z^{-1}\right), \quad z \rightarrow \infty
$$

so that for $n \geq 2$, all $K_{n}$ take the same limit at $\infty$. Thus we can define the value $\lambda_{0}$ by

$$
\lambda_{0}=\lim _{z \rightarrow \infty} K_{n}(z)=\lim _{z \rightarrow \infty} K_{2}(z)=\lim _{z \rightarrow \infty} \frac{\left(-\bar{\delta}_{1} z+\bar{\epsilon}\right) \kappa}{\bar{\delta}_{1} z+\bar{\epsilon}}=-\kappa, \quad n \geq 2 .
$$

Since also $K_{1}=-\kappa\left(\right.$ note $\left.K_{0}=\kappa\right)$, we have

$$
\lim _{z \rightarrow \infty} K_{n}(z)=\lambda_{0}=-\kappa, \quad n=1,2,3, \ldots
$$

In other words, if the formal expansion at infinity of the approximants $K_{n}$ is given by

$$
K_{n}(z)=\lambda_{0}^{(n)}+\frac{\lambda_{1}^{(n)}}{z}+\frac{\lambda_{2}^{(0)}}{z^{2}}+\cdots
$$

then $\lambda_{0}=\lambda_{0}^{(n)}=-\kappa$ for all $n \geq 1$.

Lemma 4.1 Given the point set $\alpha$, with $\alpha_{n} \neq 0$ for $n \geq 1$, the linear functional $M$, and the value of $\lambda_{0}=\kappa$, then we can uniquely define the formal Newton series $\Xi$ and $\Gamma$ as in (4.29) and (4.30).

Let us couple the data $\lambda_{0}$ and $\left\{\xi_{k}\right\}_{k \geq 0}$ with the points at which they give information, namely

$$
\begin{aligned}
& \Delta_{0}=\left(\alpha_{0}, \xi_{0}\right), \quad \Delta_{1}=\left(\hat{\alpha}_{0}, \lambda_{0}\right), \\
& \Delta_{4 n-2}=\left(\alpha_{n}, \xi_{4 n-3}\right), \quad \Delta_{4 n-1}=\left(\hat{\alpha}_{n}, \xi_{4 n-2}\right), \quad n=1,2, \ldots \\
& \Delta_{4 n}=\left(\alpha_{n}, \xi_{4 n-1}\right), \quad \Delta_{4 n+1}=\left(\hat{\alpha}_{n}, \xi_{4 n}\right), \quad n=1,2, \ldots
\end{aligned}
$$

and let us set $\Delta=\left(\Delta_{1}, \Delta_{2}, \ldots\right)$.

Since $\xi_{k}^{(2 n)}=\xi_{k}$ for $k=0,1, \ldots, 2 n-1$ and $\lambda_{0}^{(2 n)}=\lambda_{0}$, the approximant $K_{2 n}$ interpolates the data $\Delta$ up to $\Delta_{2 n}$ which are $2 n+1$ interpolation conditions. Because by $(4.10), K_{2 n}$ is of type $(n, n)$ and thus has $4 n+1$ degrees of freedom we say that $K_{2 n}$ is a multipoint Padé approximant (MPA) of type $(n, n)$ for these data.

For the odd approximants, we arrange the data as

$$
\begin{aligned}
& \nabla_{0}=\left(\hat{\alpha}_{0}, \lambda_{0}\right), \quad \nabla_{1}=\left(\alpha_{0}, \gamma_{0}\right), \\
& \nabla_{4 n-2}=\left(\hat{\alpha}_{n}, \gamma_{4 n-2}\right), \quad \nabla_{4 n-1}=\left(\alpha_{n}, \gamma_{4 n-1}\right), \quad n=1,2, \ldots \\
& \nabla_{4 n}=\left(\hat{\alpha}_{n}, \gamma_{4 n}\right), \quad \nabla_{4 n+1}=\left(\alpha_{n}, \gamma_{4 n+1}\right), \quad n=1,2, \ldots
\end{aligned}
$$

and set $\nabla=\left(\nabla_{0}, \nabla_{1}, \nabla_{2}, \ldots\right)$. For reasons similar to the ones used in the case of the even approximants, we see that $K_{2 n+1}$ interpolates the data $\nabla$ up to $\nabla_{2 n}$ which are $2 n+1$ 
interpolation conditions. Because $K_{2 n+1}$ is of type $(n, n)$, we can say that $K_{2 n+1}$ is a MPA of type $(n, n)$ for the data $\nabla$.

Since

$$
K_{2 n+1}-\Xi=O\left(V_{2 n+1}\right)
$$

and since by (4.22) and (4.24), we have for the Newton expansions with respect to the basis $V_{k}$ that

$$
K_{2 n+1}-K_{2 n+2}=O\left(V_{2 n-1}\right), \quad n \geq 1,
$$

it follows that $\xi_{k}^{(2 n+1)}=\xi_{k}$ for $k=0,1, \ldots, 2 n-2$. Thus we see that $K_{2 n+1}$ interpolates the data $\Delta$ up to $\Delta_{2 n-1}$ which are $2 n$ conditions. Because $K_{2 n+1}$ is of type $(n, n)$, this is one condition short to be called an MPA for the data $\Delta$.

By similar arguments one can obtain that the approximants $K_{2 n}$ interpolate the data $\nabla$ up to $\nabla_{2 n-1}$, which are again $2 n$ interpolation conditions. Because also $K_{2 n}$ is of type $(n, n)$, once more we are one interpolation condition short to call $K_{2 n}$ a MPA for the data $\nabla$.

Theorem 4.2 Suppose $\alpha_{n} \neq 0$ for $n \geq 1$. Suppose the interpolation data $\Delta$ and $\nabla$ are defined as in (4.32)-(4.34) and (4.35)-(4.37). Then $K_{2 n}$ is a MPA for $\Delta$ of type $(n, n)$ and it interpolates the first $2 n$ data couples from $\nabla$. On the other hand $K_{2 n+1}$ is a MPA for $\nabla$ of type $(n, n)$ and it interpolates the first $2 n$ data couples of $\Delta$.

So far, we have assumed that all $\alpha_{n} \neq 0$ for $n \geq 1$. Now assume that in the sequence $\left\{\alpha_{1}, \alpha_{2}, \ldots, \alpha_{k}\right\}$ we have $\alpha_{i}=0$ for $m$ different values of $i$. Then of course the corresponding factor $\left(1-\bar{\alpha}_{i} z\right)=1$. Taking this into account when inspecting the right-hand sides of $(4.21)-(4.24)$, it follows that

$$
K_{n+1}(z)-K_{n}=O\left(z^{-m^{\prime}}\right), \quad \text { with } m^{\prime} \geq 2 m+1 \text { for } n \geq 4 k+1 .
$$

Thus there must exist numbers $\lambda_{0}, \ldots, \lambda_{2 m}$, independent of $n$ such that

$$
K_{n}(z)=\lambda_{0}+\lambda_{1} z^{-1}+\cdots+\lambda_{2 m} z^{-2 m}+\lambda_{2 m+1}^{(n)} z^{1-2 m}+\cdots, \quad z \rightarrow \infty, \quad \forall n \geq 4 m+1 .
$$

Thus if $\alpha_{k}=0$ for $0<k=n_{1}, n_{2}, \ldots, n_{m}$ with $m \leq \infty$, then we can define a polynomial of degree $2 m$ if $m$ is finite, or a formal power series if $m$ is infinite, say

$$
L(z)=\lambda_{0}+\frac{\lambda_{1}}{z}+\frac{\lambda_{2}}{z^{2}}+\cdots+\frac{\lambda_{2 m}}{z^{2 m}}
$$

Although checking all the details is a tedious job, one can verify that the following procedure will allow us to keep the interpolation properties of the approximants as given in Theorem 4.2 without the condition that the $\alpha_{n} \neq 0$ for $n \geq 1$.

0 . Suppose $\alpha_{k}=0$ for $k \in\left\{n_{1}, n_{2}, \ldots\right\}$ with $0<n_{1}<n_{2}<\cdots$.

1. Then the set $\left\{V_{n}\right\}_{n=0}^{\infty}$ will not be a basis for $\Pi$. To turn this into a basis, drop all the vectors $V_{4 n_{i}-1}$ and $V_{4 n_{i}+1}, i=1,2, \ldots$. Similarly turn $\left\{W_{n}\right\}_{n=0}^{\infty}$ into a basis by dropping the vectors $W_{4 n_{i}-2}$ and $W_{4 n_{i}}$.

2. By this operation, the coefficients $\xi_{4 n_{i}-1}, \xi_{4 n_{i}+1}, \gamma_{4 n_{i}-2}$ and $\gamma_{4 n_{i}}$ will disappear as well. Replace in the data set $\Delta$ the coefficients $\xi_{4 n_{i}-1}$ and $\xi_{4 n_{i}+1}$ by $\lambda_{i}$, and replace in the data set $\nabla$ the coefficients $\gamma_{4 n_{i}-2}$ and $\gamma_{4 n_{i}}$ by $\lambda_{i}$ 
Interpolation of the data set $\Delta$ then means that the approximant will match a number of coefficients in the formal Newton series $\Xi$ and a number of terms in the formal power series $L$ at $\infty$. If all $\alpha_{n} \neq 0$ for $n \geq 1$, there is only one coefficient, namely $\lambda_{0}$, in the series $L$ defined, and this one coefficient is matched. All the other interpolation effort goes to the series $\Xi$. However, each time a data couple is met with $\hat{\alpha}_{n_{i}}=0$, then a coefficient in $\Xi$ is skipped and a coefficient in $L$ is matched instead. A similar interpretation can be given for interpolation of the data set $\nabla$.

In the special case where all $\alpha_{k}=0$, then $\Xi=\Gamma$ will be formal power series at the origin and $L$ will be a formal power series at $\infty$. The approximants are then two-point Padé approximants (2PA) for $\Gamma$ and $L$. The even approximants $K_{2 n}$ match $n+1$ terms of $\Gamma$ and $n$ terms of $L$ while the odd approximants match $n+1$ terms of $L$ and $n$ terms of $\Gamma$. They are the the $2 \mathrm{PAs}$ in the balanced situation.

\section{References}

[1] A. Bultheel. Laurent series and their Padé approximations, volume OT-27 of Oper. Theory: Adv. Appl. Birkhäuser Verlag, Basel-Boston, 1987.

[2] A. Bultheel and P. Dewilde. Orthogonal functions related to the Nevanlinna-Pick problem. In P. Dewilde, editor, Proc. 4th Int. Conf. on Math. Theory of Networks and Systems at Delft, pages 207-212, North-Hollywood, 1979. Western Periodicals.

[3] A. Bultheel, P. González-Vera, E. Hendriksen, and O. Njåstad. The computation of orthogonal rational functions and their interpolating properties. Numer. Algorithms, 2(1):85-114, 1992.

[4] A. Bultheel, P. González-Vera, E. Hendriksen, and O. Njåstad. Moment problems and orthogonal functions. J. Comput. Appl. Math., 48:49-68, 1993.

[5] A. Bultheel, P. González-Vera, E. Hendriksen, and O. Njåstad. Orthogonal rational functions on the unit circle: theory, applications and generalizations. In M. Alfaro, A. García, C. Jagels, and F. Marcellan, editors, Orthogonal Polynomials on the Unit Circle: Theory and Applications, pages 21-42, 1994.

[6] A. Bultheel, P. González-Vera, E. Hendriksen, and O. Njåstad. Quadrature formulas on the unit circle based on rational functions. J. Comput. Appl. Math., 50:159-170, 1994.

[7] A. Bultheel, P. González-Vera, E. Hendriksen, and O. Njåstad. Recurrence relations for orthogonal functions. In S.C. Cooper and W.J. Thron, editors, Continued Fractions and Orthogonal Functions, volume 154 of Lecture Notes in Pure and Appl. Math., pages 24-46. Marcel Dekker, 1994.

[8] M.M. Djrbashian. A survey on the theory of orthogonal systems and some open problems. In P. Nevai, editor, Orthogonal polynomials: Theory and practice, volume 294 of Series C: Mathematical and Physical Sciences, pages 135-146, Boston, 1990. NATOASI, Kluwer Academic Publishers.

[9] W.B. Jones and W.J. Thron. Continued Fractions. Analytic Theory and Applications. Addison-Wesley, Reading, Mass., 1980. 
[10] X. Li and K. Pan. Strong and weak convergence of rational functions orthogonal on the unit circle. J. London Math. Soc., 53:289-301, 1996.

[11] G.L. López. Szegő's theorem for orthogonal polynomials with respect to varying measures. In M. Alfaro et al., editors, Orthogonal polynomials and their applications, volume 1329 of Lecture Notes in Math., pages 255-260. Springer, 1988.

[12] G.L. López. Asymptotics of polynomials orthogonal with respect to varying measures. Constr. Approx., 5:199-219, 1989.

[13] L. Lorentzen and H. Waadeland. Continued fractions with applications, volume 3 of Studies in Computational Mathematics. North-Holland, 1992.

[14] K. Pan. On orthogonal systems of rational functions on the unit circle and polynomials orthogonal with respect to varying measures. J. Comput. Appl. Math., 47(3):313-322, 1993.

[15] K. Pan. Extensions of Szegö's theory of rational functions orthogonal on the unit circle. J. Comput. Appl. Math., 62:321-331, 1995.

[16] W.J. Thron. L-polynomials orthogonal on the unit circle. In A.M. Cuyt, editor, Nonlinear Numerical Methods and Rational Approximation, pages 271-278. Kluwer, 1988. 